\title{
A Dynamic Model for the Analysis of Surge in a Tropical Storm
}

\author{
Alexis Amézaga-Hechavarría ${ }^{1}$, José Marín-Antuña ${ }^{2}$, Manuel Tejera-Fernández ${ }^{3}$ and \\ Julio Navarro-Serrano ${ }^{4}$ \\ ${ }^{1}$ Group of Theoretical Physics, ICIMAF \\ Calle E \# 309, esq. a 15, CP 10400 Ciudad Habana, Cuba, \\ ${ }^{2}$ Faculty of Physics, University of Havana \\ San Lázaro y L, Habana, Cuba, \\ ${ }^{3}$ Department of Physics, IPSJAE, Marianao, Habana, Cuba, \\ ${ }^{4}$ Department of Nautic Research, GEOCUBA, Calle 4, \# 304, Playa, Habana. \\ Received 12 November, 1998; Revised 22 March, 1999
}

\begin{abstract}
We obtain an analytic representation for the profile of the surge wave in the sea, due to a tropical storm at a low latitude. The results reproduce the known experimental data adequately. In the calculations we take into account the influence of Rossby's second parameter and we analyse its contribution to the surge wave, which is proved to be sufficiently small to be neglected in higher latitudes. The obtained analytical solution allows us to study in detail the surge wave formation in the presence of such a storm. To solve the problem, Laplace method and Green functions are used. This allows us to improve previous results obtained by Evsa in 1989.
\end{abstract}

\section{Introduction}

In the last years the interest of studying non-periodic oscillations of sea level has grown. In particular, it is of interest the study of those oscillations excited by pressure fields and strong winds in the tropical storm. It is possible to find previous information about this theme in the work of Jelesniansky [1] and more recently, Johns et al. [2], Fandry et al. [3], Jerome et al. [4], Signorini [5], Davies et al. [6] and Lardner et al. [7] and [8]. The majority of these papers employ numerical methods or qualitative analysis of the solution of the mathematical model. In the present paper we obtain an analytic solution of the problem, similar to the one formulated by Evsa [9]. In this work, there is an incorrect expresion for the Green function of the problem. Therefore, for the analytic solution, Evsa does not take account of the multi-valuated character of the complex variable function in the path integrations. In the present paper we make correct use of the Green function method and the integral transform techniques. We also analyze the influence of Rossby's second parameter, which is usually neglected at high latitudes by other authors because of its smallness with respect to Rossby's first parameter and because it allows to simplify the calculation. We check that Rossby's second parameter is small enough to be neglected at low latitudes.

\section{Formulation of the problem}

Let us consider a tropical cyclone (TC) which causes a perturbation of the sea surface in an ocean region distant from any coast. The interaction region has a characteristic deepness less than the storm horizontal size by two orders of magnitude. Therefore, we can use the shalow water approximation. We also consider a quasistationary regime for the storm, in order to keep a fixed coordinate center. Due to the axial symmetry of this problem, we use a cylindrical system of coordinates with center on the axis of the cyclone.

The Navier-Stokes equations in the cilindrical system of coordinates including the Coriolis terms are given by

$$
\frac{\partial u}{\partial t}+u \frac{\partial u}{\partial r}+\frac{v}{r} \frac{\partial u}{\partial \varphi}+w \frac{\partial u}{\partial z}-\frac{v^{2}}{r}=f v-f^{*} w-\frac{1}{\rho} \frac{\partial P_{a}}{\partial r}+\frac{1}{\rho} \frac{\partial \sigma_{r z}}{\partial z}
$$




$$
\begin{gathered}
\frac{\partial v}{\partial t}+u \frac{\partial v}{\partial r}+\frac{v}{r} \frac{\partial v}{\partial \varphi}+w \frac{\partial v}{\partial z}-\frac{u v}{r}=-f u-\frac{1}{\rho} \frac{1}{r} \frac{\partial P_{a}}{\partial \varphi}+\frac{1}{\rho} \frac{\partial \sigma_{\varphi z}}{\partial z}, \\
\frac{\partial w}{\partial t}+u \frac{\partial w}{\partial r}+\frac{v}{r} \frac{\partial w}{\partial \varphi}+w \frac{\partial w}{\partial z}=f^{*} u-\frac{1}{\rho} \frac{\partial P_{a}}{\partial z}-g
\end{gathered}
$$

with $u=v_{r}, v=v_{\varphi}, w=v_{z}, f=2 \Omega \sin \phi, f^{*}=$ $2 \Omega \cos \phi$. Here $\Omega$ is the angular Earth velocity and $\phi$ is the latitude of the region under consideration.

In these equations the term of the divergence of the stress tensor contains only the vertical stress forces because they are much larger than the rest of the internal friction effects. In equation (3), all the stress effects are neglected in comparison with the vertical pressure gradient and with the gravity acceleration.

The equation of continuity in cylindrical coordinates is

$$
\frac{\partial u}{\partial r}+\frac{1}{r} \frac{\partial v}{\partial \varphi}+\frac{\partial w}{\partial z}+\frac{u}{r}=0 .
$$

The bondary conditions are: in the botton, the adherence condition; at the sea surface, the kinematic condition $\left(\left.w\right|_{z=\xi}=\frac{d \xi}{d t} \equiv u \frac{\partial \xi}{\partial r}+\frac{v}{r} \frac{\partial \xi}{\partial \varphi}+\frac{\partial \xi}{\partial t}\right)$.

The $\mathrm{Oz}$ axis coincides with the external normal vector to the Earth surface. We also suppose the integration time in these equations is much smaller than the cyclone displacement characteristic time. Therefore, we take into account the time derivatives, although we consider the TC static.

By integration of equation (1)-(3) along the $\mathrm{z}$ direction, we obtain the following system, for the shallow water approximation, in dimensionless form,

$$
\begin{aligned}
& S t^{-1} \frac{\partial u}{\partial t}+(\xi+h) \frac{\partial P_{a}}{\partial r}+F r^{-1}(\xi+h) \frac{\partial \xi}{\partial r}-R o^{-1} v+R o^{*-1} \int_{-h}^{\xi} w d z=\tau_{r}^{s}-\tau_{r}^{f} \\
& S t^{-1} \frac{\partial v}{\partial t}+\frac{(\xi+h)}{r} \frac{\partial P_{a}}{\partial \varphi}+F r^{-1} \frac{(\xi+h)}{r} \frac{\partial \xi}{\partial \varphi}+R o^{-1} u=\tau_{\varphi}^{s}-\tau_{\varphi}^{f} \\
& S t^{-1} \frac{\partial \xi}{\partial t}+\frac{\partial u}{\partial r}+\frac{1}{r} \frac{\partial v}{\partial \varphi}+\frac{u}{r}=0 .
\end{aligned}
$$

We neglect the convective acceleration terms, because the absence of coasts implies that the velocity gradients are very small in the considered region. In the above equations $\xi$ is the sea perturbation average level; $h$ is the non-perturbed sea deepness; $P_{a}$ is the atmospheric pressure field originated by the TC; $\tau_{i}^{s}$ are the surface stresses produced by the TC winds and $\tau_{i}^{s}$ are the bottom stresses $(i=r, \varphi) ; \rho_{0}$ is the water density; $H$ is the characteristic deepness of the region; $S t=\left(U_{0} T\right) / L$ is the Struhal's number; $F r=U_{0}^{2} /(g H)$ is Froude's number; $R_{0}=U_{0} /(f L)$ is Rossby's first parameter and $R_{0}^{*}=U_{0} /\left(f^{*} H\right)$ is Rossby's second parameter; $U_{0}$ is the characteristic velocity; $L$ is the characteristic size on the horizontal ocean surface plane. We take $\Phi \approx 5^{\circ}$ because the contribution of $f^{*}$ is bigger at very low latitudes. This is precisely the interest of our study, maintaining $f$ not equal to zero in the model.

In the following calculation we neglect $\tau_{i}^{b}$ because the processes are essentially superficial and therefore the bottom does not matter. Also, we consider $\xi+h \approx$ $h=1$ because in the problem $\xi \ll h$ and that the problem is axisymmetric so that $\frac{\partial[. .]}{\partial \varphi} \equiv 0$. The presence of Rossby's second parameter in equation (5) makes it impossible to use the two-dimensional approach employed by other authors. To avoid this problem, we assume an exponential dependence of the horizontal velocities with respect to the vertical coordinate,

$$
\begin{aligned}
& u(r, \varphi, z, t)=u_{0}(r, \varphi, t) \exp [-a(\xi-z)], \\
& v(r, \varphi, z, t)=v_{0}(r, \varphi, t) \exp [-a(\xi-z)] .
\end{aligned}
$$


We take an exponential dependence because, in many fluid problems, that is the law of variation of the horizontal velocities with respect to the deepness and considering the gravity constant in the studied region. This assumption is consistent with our model, in which $\mathrm{Fr}$ is constant.

With this dependence we have that, at the surface $z=\xi$, velocities are $u_{0}$ and $v_{0}$. The parameter $a$ controls the decrease of the velocities with $z$. Expressions (8), using (4), allow us to obtain

$$
\int_{-h}^{\xi} w d z=H\left(u \frac{\partial \xi}{\partial r}+\frac{v}{r} \frac{\partial \xi}{\partial \varphi}\right)-\frac{H}{a}\left(\frac{\partial u}{\partial r}+\frac{1}{r} \frac{\partial v}{\partial \varphi}+\frac{u}{r}\right) \equiv \Pi
$$

We have neglected the convective derivative of $\xi$ in the first term because the calculations are made in open sea [10], [11] and also because we only consider the long wave generated by TC. Hence $(H=1$, the dimensionless formulation)

$$
\Pi=\frac{1}{a}\left(\frac{\partial u}{\partial r}+\frac{u}{r}\right) \cdot 1
$$

Then, system (1) - (3) takes the form

$$
\begin{aligned}
& S t^{-1} \frac{\partial u}{\partial t}+\frac{\partial P a}{\partial r}+F r^{-1} \frac{\partial \xi}{\partial r}-R o^{-1} v+R o^{*-1} \Pi=\tau_{r}^{s} \\
& S t^{-1} \frac{\partial v}{\partial t}+R o^{-1} u=\tau_{\varphi}^{s} \\
& S t^{-1} \frac{\partial \xi}{\partial t}+\frac{\partial u}{\partial r}+\frac{u}{r}=0 .
\end{aligned}
$$

To solve this system we consider homogeneous initial conditions and the following boundary conditions,

$$
\left.u\right|_{r \rightarrow \infty}=\left.v\right|_{r \rightarrow \infty}=\left.\xi\right|_{r \rightarrow \infty}=0,\left.\quad \xi\right|_{r=0}<M
$$

To accomplish the calculations we take, following Johns et al. [2], $\tau_{r}^{s}=\tau_{0} \sin \alpha \beta^{2}(r), \tau_{\varphi}^{s}=\tau_{0} \cos \alpha \beta^{2}(r)$, where $\tau_{0}=\rho_{1} \gamma_{1}^{2} W_{0}^{2}$ and $\beta(r)=2(r / R) /\left[1+(r / R)^{2}\right]$. Here $\rho_{1}$ is the density of the air, $\gamma_{1}$ is the drag coefficient, $W_{O}$ is the wind velocity in the radius $R$ of the greatest winds, and $\alpha$ is the fluency angle of the wind in the TC. We take the following dependence for the atmospheric pressure $P a(r)=P_{\infty}-\Delta P /\left[1+(r / R)^{2}\right]^{1 / 2}$ in correspondence with the common assumption in the studies of surge in tropical storms (see, for instance, $[1,11])$. tion $u, v, \xi$ (see e.g. [12]),

$$
\begin{aligned}
& u(r, \varphi, t)=\sum_{n=0}^{\infty} u_{n}(r, \varphi, t) \varepsilon^{n}, \\
& v(r, \varphi, t)=\sum_{n=0}^{\infty} v_{n}(r, \varphi, t) \varepsilon^{n}, \\
& \xi(r, \varphi, t)=\sum_{n=0}^{\infty} \xi_{n}(r, \varphi, t) \varepsilon^{n} .
\end{aligned}
$$

Substituting (15) - (16) into equations (11) - (13) and taking only the two first terms of the sequence $u_{0}$, $v_{0}, \xi_{0}$, and $u_{1}, v_{1}, \xi_{1}$, we obtain the following systems:

\section{Calculation}

In the system of equations (11)-(13), $\varepsilon \equiv\left(R o^{*}\right)^{-1} \ll 1$. Hence, we assume an analytic dependence of the solu-

\footnotetext{
${ }^{1}$ If directly we shall take a linear variation of the vertical velocity, for instance, $w=(1+z / H)(d \xi / d t)$, it simply shall give another numerical value of the coefficient in (10).
} 
-Zero-order system

$$
\begin{aligned}
& S t^{-1} \frac{\partial u_{0}}{\partial t}+\frac{\partial P a}{\partial r}+F r^{-1} \frac{\partial \xi_{0}}{\partial r}-R o^{-1} v_{0}=\tau_{r}^{s}, \\
& S t^{-1} \frac{\partial v_{0}}{\partial t}+R o^{-1} u_{0}=\tau_{\varphi}^{s}, \\
& S t^{-1} \frac{\partial \xi_{0}}{\partial t}+\frac{\partial u_{0}}{\partial r}+\frac{u_{0}}{r}=0 ;
\end{aligned}
$$

-First-order system.

$$
\begin{aligned}
& S t^{-1} \frac{\partial u_{1}}{\partial t}+\xi_{1} \frac{\partial P a}{\partial r}+F r^{-1} \frac{\partial \xi_{1}}{\partial r}-R o^{-1} v_{1}+\Pi_{0}=0, \\
& S t^{-1} \frac{\partial v_{1}}{\partial t}+R o^{-1} u_{1}=0, \\
& S t^{-1} \frac{\partial \xi_{1}}{\partial t}+\frac{\partial u_{1}}{\partial r}+\frac{u_{1}}{r}=0 .
\end{aligned}
$$

In our case, it is sufficient to take the solution at first order in perturbation theory, because the small parameter $\varepsilon$ in (11) is multipliying a term which is of a very small order, due to the smoothness of the used model. This fact leads to a non-singular perturbation problem. Hence, $(15)-(17)$ are, not only asymptotic, but also monotonic expansions. We apply the Laplace transform to system $(18)-(20)$. For $E_{0}(r, p)=L\left\{\xi_{0}(r, t)\right\}$ we obtain the following equation

$$
\frac{1}{r} \frac{d}{d r}\left[r \frac{d E_{0}}{d r}\right]-R_{p} E_{o}=F r\left[\frac{\partial F(r, p)}{\partial r}-\frac{F(r, p)}{r}\right],
$$

where $F(r, p)=F_{1}(r, p)-(S t / p R o) F_{2}(r, p), F_{1}(r, p)=\left[\tau_{0} \sin \alpha \beta^{2}(r)-\partial P a / \partial r\right] / p, F_{2}(r, p)=\tau_{0} \cos \alpha \beta^{2}(r) / p$, $R_{p}=\delta\left(p^{2}+\theta^{2}\right), \delta=F r / S t^{2}, \theta=S t / R o$ and $p=s+i \sigma$.

We can express the solution of equation (24) as

$$
E_{0}(r, p)=F r \int_{0}^{\infty} G(r, s)\left[\frac{\partial F(s, p)}{\partial s}-\frac{F(s, p)}{s}\right] d s,
$$

where

$$
G(r, s)=\left\{\begin{array}{l}
\frac{I_{0}\left(\sqrt{R_{p}} r\right)}{I_{0}\left(\sqrt{R_{p}} R_{1}\right)}\left[I_{0}\left(\sqrt{R_{p}} R_{1}\right) K_{0}\left(\sqrt{R_{p}} s\right)-I_{0}\left(\sqrt{R_{p}} s\right) K_{0}\left(\sqrt{R_{p}} R_{1}\right)\right] \\
0 \leq r \leq s<R_{1} \\
\frac{I_{0}\left(\sqrt{R_{p}} s\right)}{I_{0}\left(\sqrt{R_{p}} R_{1}\right)}\left[I_{0}\left(\sqrt{R_{p}} R_{1}\right) K_{0}\left(\sqrt{R_{p}} r\right)-I_{0}\left(\sqrt{R_{p}} r\right) K_{0}\left(\sqrt{R_{p}} R_{1}\right)\right] \\
0 \leq s \leq r<R_{1}
\end{array}\right.
$$

is the Green function of the operator of equation (24), which satisfies the boundary conditions $\left.G(r, s)\right|_{r=0}<M$; $\left.G(r, s)\right|_{r \rightarrow \infty}=0 ; R_{1} ; R_{1}$ is the TC external radius.

The inverse Laplace transform gives

$$
\begin{aligned}
\xi_{0}(r, t) & =\int_{0}^{r} \frac{1}{2 \pi i} \int_{a-i \infty}^{a+i \infty} G(r, s)\left[\frac{A_{1}(s)}{p}+A_{2}(s) p^{2}\right] \exp (p t) d p d s \\
& +\int_{r}^{R_{1}} \frac{1}{2 \pi i} \int_{a-i \infty}^{a+i \infty} G(r, s)\left[\frac{A_{1}(s)}{p}+A_{2}(s) p^{2}\right] \exp (p t) d p d s
\end{aligned}
$$

with 


$$
\begin{aligned}
& A_{1}(s)=F r \tau_{0} \sin \alpha \beta(s)[2 \partial \beta(s) / \partial s-\beta(s) / s]+\left[\partial^{2} P a / \partial s^{2}-\partial P a / \partial s\right], \\
& A_{2}(s)=\left(F r S t / R_{0}\right) \tau_{0} \cos \alpha \beta(s)[\beta(s) / s-2 \partial \beta(s) / \partial s] .
\end{aligned}
$$

Usual techniques of the theory of functions of complex variables allow us to calculate the contour integrals in (27). We obtain

$$
\xi_{0}(r, t)=\frac{2}{p i} \sum_{i=1}^{4} I_{i}
$$

where

$$
\begin{aligned}
& I_{1}=\int_{0}^{r} \int_{0}^{\theta} G(r, s) \frac{A_{1}(s) \sin (\rho t)}{\rho} d \rho d s, \\
& I_{2}=\int_{0}^{r} \int_{0}^{\theta} G(r, s) \frac{A_{2}(s)(1-\cos (\rho t)}{\rho^{2}} d \rho d s, \\
& I_{3}=\int_{r}^{R_{1}} \int_{0}^{\theta} G(r, s) \frac{A_{1}(s) \sin (\rho t)}{\rho} d \rho d s, \\
& I_{4}=\int_{r}^{R_{1}} \int_{0}^{\theta} G(r, s) \frac{A_{2}(s)(1-\cos (\rho t)}{\rho^{2}} d \rho d s .
\end{aligned}
$$

In similar form we apply the Laplace transform to system (21)-(23) and obtain for $E_{1}(r, p)=L\left\{\xi_{1}(r, t)\right\}$ the equation

$$
\frac{1}{r} \frac{d}{d r}\left[r \frac{d E_{1}}{d r}\right]-R_{p} E_{1}=F_{r}\left[\frac{\partial F(r, p)}{\partial r}-F(r, p)\right],
$$

where

$$
F(r, p)=\frac{p E_{0}(r, p)}{a S t}
$$

The solution of (34) is

$$
E_{1}(r, p)=F r \int_{0}^{R_{1}} G(r, s)\left[\frac{\partial F(s, p)}{\partial s}-F(s, p)\right] d s .
$$

We proceeded as in the above zero order case in order to obtain $\xi_{1}(r, t)$. The results are

$$
\xi_{1}(r, t)=\frac{2}{\pi} \sum_{j=1}^{4} \sum_{i=1}^{2}\left(A_{i}^{j}+B_{i}^{j}\right),
$$

where $A_{i}^{j}$ and $B_{i}^{j}$ are given in the appendix.

\section{Results and Discussion}

We have obtained an analytic representation for the profile of the surge wave in the sea due to a TC at low latitude, given by

$$
\xi(r, t)=\xi_{0}(r, t)+\varepsilon \xi_{1}(r, t),
$$

where $\xi_{0}$ and $\xi_{1}$ are given by (29) and (37), respectively.

As it can be seen, these expresions present diffi- culties to analyse the main properties of the solution. However, they allow checking the analitical dependence with respect to model input parameters. The obtained dependence shows that the expression is stable with respect to any continued variation of the corresponding parameter.

We have numerically calculated the integrals involved in (38) in order to verify the correspondence 
between the obtained solution and the experimental data. The values of the parameters are the following: $t=0.5,1, \ldots, 4$ hours, $R=50 \mathrm{Km}, W_{0}=38 \mathrm{~m} / \mathrm{s}, \gamma_{1}=$ $0.0025, \Delta_{p}=55 \mathrm{mBar}, \alpha=10^{\circ}, \rho=1.293 \mathrm{Kg} / \mathrm{m}^{3}$, $S t=0.01, R_{0}=0.79, \epsilon=0.14$ and $F r=0.0001$. We elaborate graphs of sea excitation vs. TC radius.

From Figs. 1, 2 and 3, it can be seen the dynamics of the surge wave formation. The plots show that the solution qualitatively maintains its form during time and that it smooth.

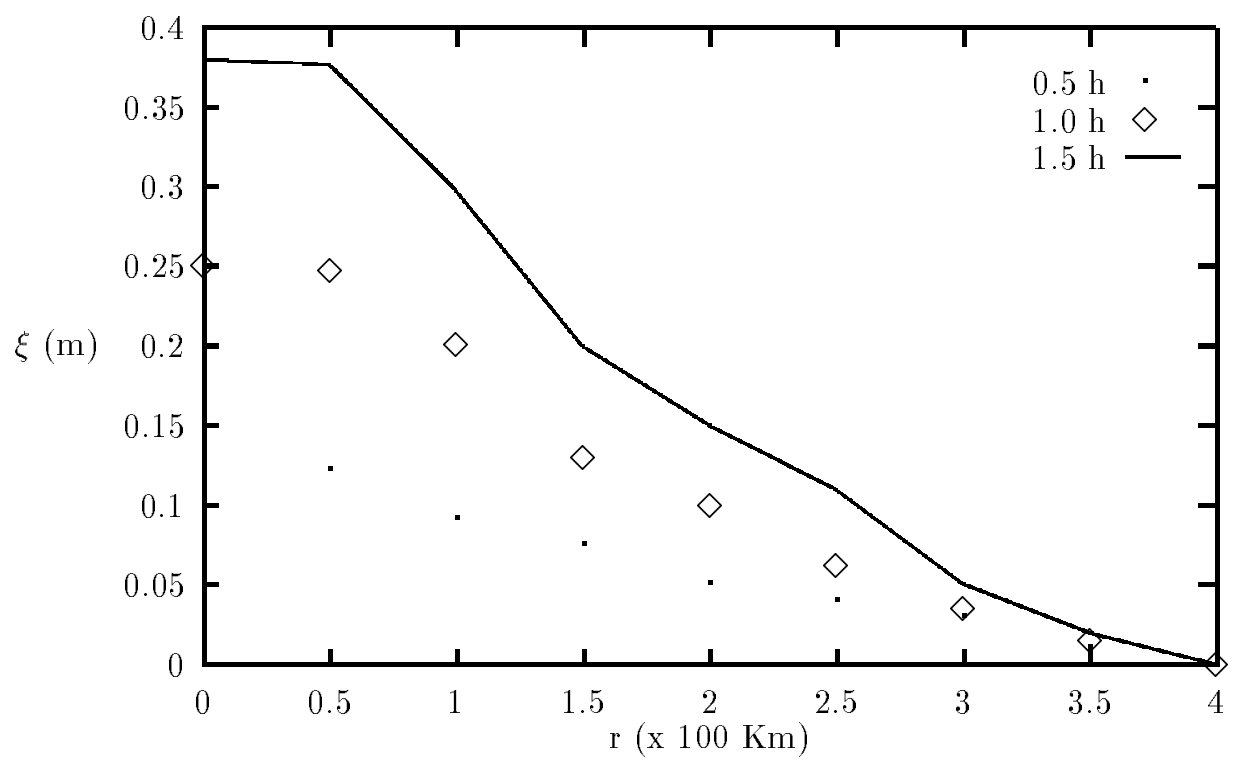

Figure 1. Profiles of the surge wave for $t=0.5,1.0$ and 1.5 hours.

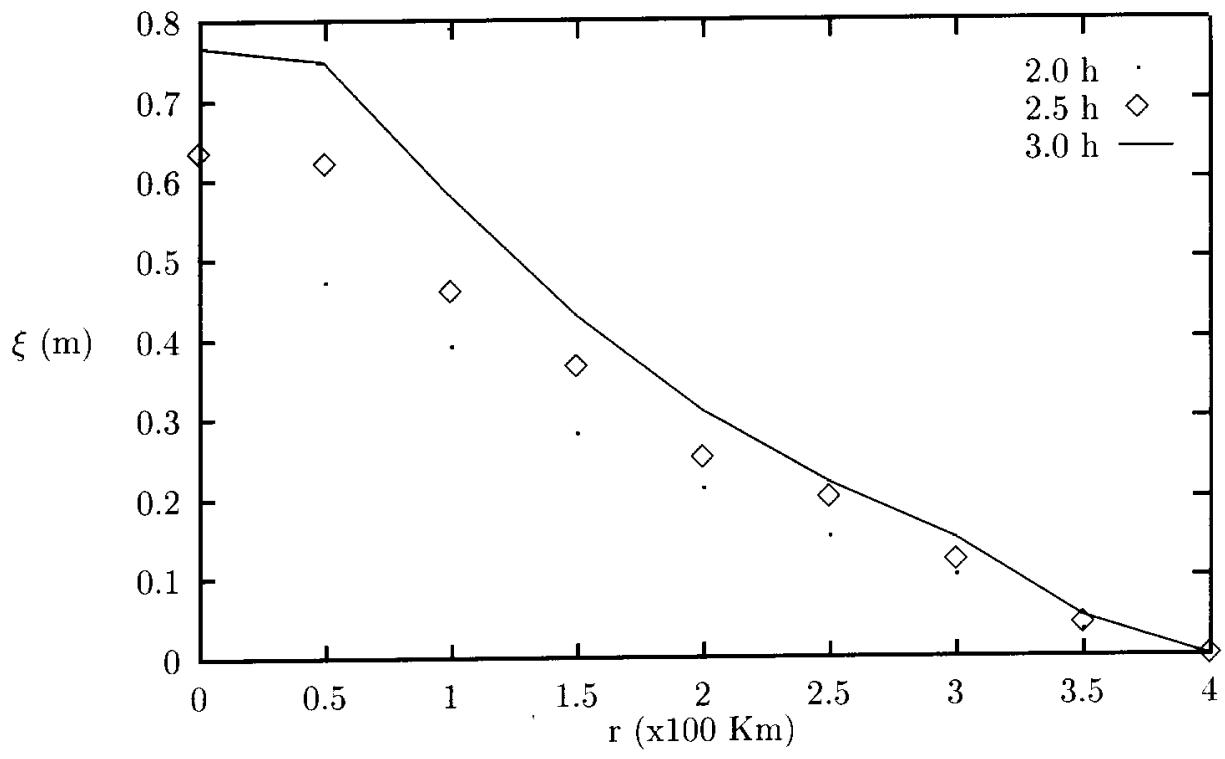

Figure 2. Profiles of the surge wave for $t=2.0,2.5$ and 3.0 hours. 


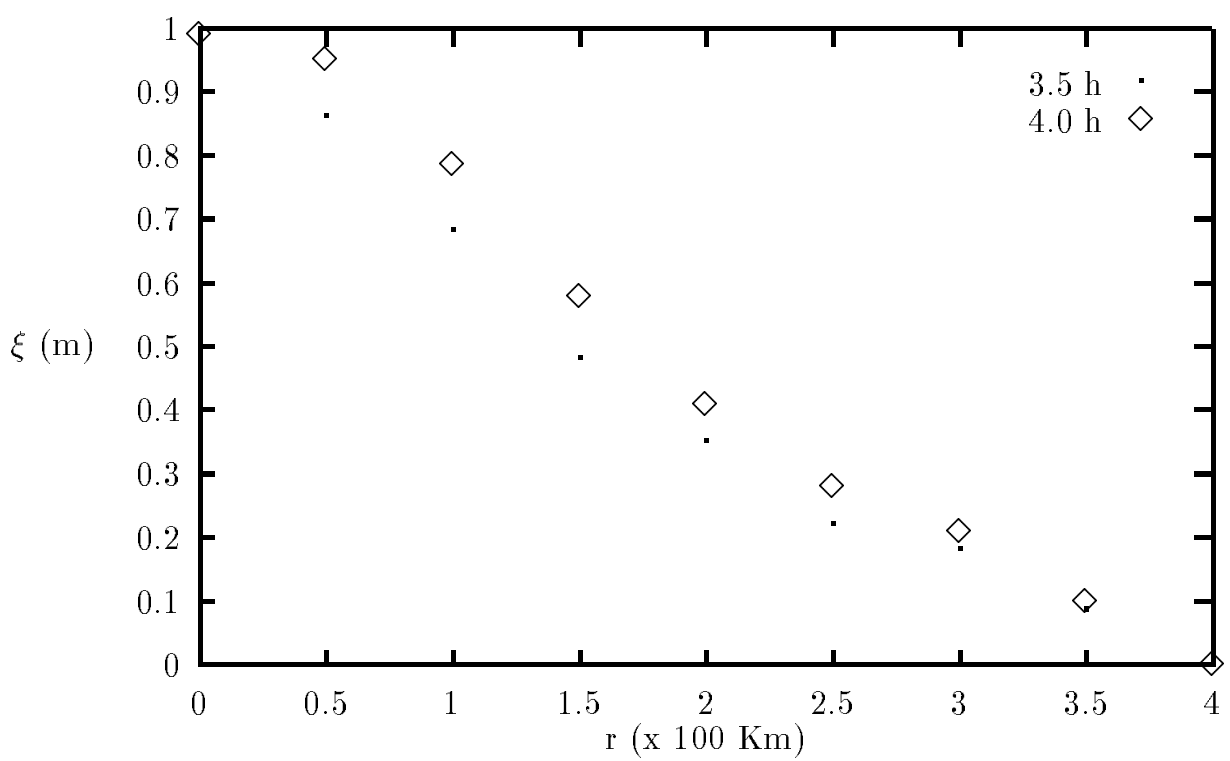

Figure 3. Profiles of the surge wave for $\mathrm{t}=3.5$ and 4.0 hours.

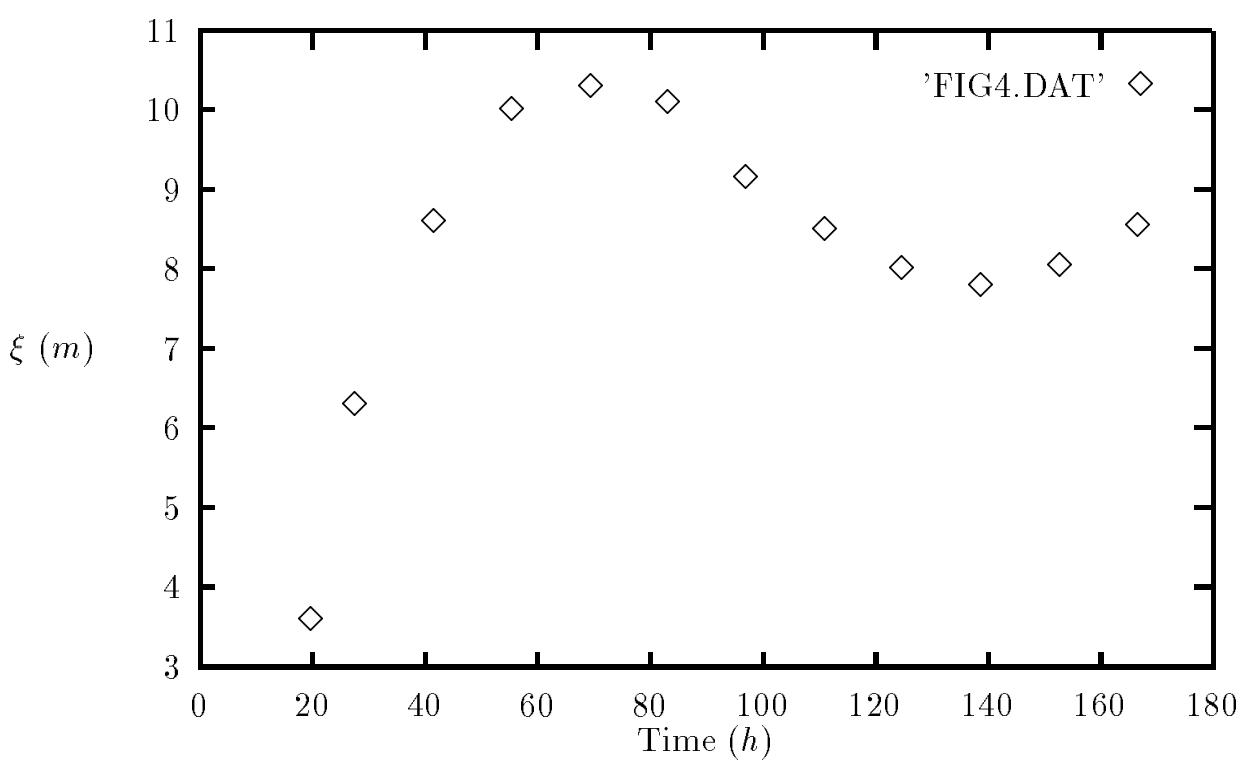

Figure 4. Evolution in time of the surface excitation in $r=0$.

Table 1. Contribution of the second Rossby parameter to the surface excitation in $r=0$ for several times.

\begin{tabular}{|c|c|c|c|c|}
\hline Excitation $(r=0)$ & $t=1 \mathrm{~h}$ & $t=2 \mathrm{~h}$ & $t=3 \mathrm{~h}$ & $t=4 \mathrm{~h}$ \\
\hline$\xi_{0}(\mathrm{~m})$ & 0.255 & 0.510 & 0.764 & 1.020 \\
\hline$\xi=\xi_{0}+\varepsilon \xi_{1}(\mathrm{~m})$ & 0.257 & 0.512 & 0.766 & 1.023 \\
\hline
\end{tabular}

In Fig. 4 a second experiment shows that the solution tends to a bounded oscillatory expression as the time $t$ increases. It is necessary to say that the limit values of the excitation in this case do not correspond to the real values, because, with the increasing of time, the validity of the quasistationary model does not hold. Nevertheless, this experiment assures that our formal solution does not diverge in time. 
In Table 1, we show the result of another experiment in which it can be seen the real contribution of Rossby's second parameter, i.e. the contribution to the surface excitation, due to the horizontal streams caused by the deflection of the vertical internal streams into the sea as a result of the Earth rotation.

It can be seen that this contribution is small, due to the fact that the vertical streams are quite small in comparison with the horizontal streams induced by the wind of the TC. Moreover, the relative increase of the term in the equation containing Rossby's second parameter with respect to the term that contains Rossby's first parameter is due to the abrupt decrease of the latter. Hence, it is possible to neglect the contribution of
Rossby's streams even at low latitudes.

We have obtained an analytical expression of the excitation of the sea level in the presence of a tropical cyclone, whose results are similar to those obtained by numerical methods. The analytical solution allows us to study in detail the surge wave formation in the presence of a tropical cyclone. It is possible to see that a non-periodical wave is obtained which tends to some stationary state. It is interesting to highlight that the contribution of Rossby's second parameter is quite small even in low latitudes. This result justifies the direct use of the two-dimensional system of order zero to calculate the surge.

\section{Appendix}

Expresions for the coeficients $A_{i}^{j}$ and $B_{i}^{j}$ in formula (37).

$$
\begin{aligned}
& A_{1}^{1}=\int_{0}^{r} \int_{0}^{s} \int_{0}^{\theta} G_{1}(s) G_{2}(r) \frac{G_{2}(s)}{s} G_{1}(y) A_{1}(y) \cos (\rho t) d \rho d y d s \\
& A_{1}^{2}=\int_{0}^{r} \int_{0}^{s} \int_{0}^{\theta} G_{1}(s) G_{2}(r) \frac{\partial G_{2}(s)}{\partial s} G_{1}(y) A_{1}(y) \cos (\rho t) d \rho d y d s \\
& A_{1}^{3}=\int_{0}^{r} \int_{s}^{R_{1}} \int_{0}^{\theta} G_{2}(r) G_{1}(s) \frac{G_{1}(s)}{s} G_{2}(y) A_{1}(y) \cos (\rho t) d \rho d y d s \\
& A_{1}^{4}=\int_{0}^{r} \int_{s}^{R_{1}} \int_{0}^{\theta} G_{2}(r) G_{1}(s) \frac{\partial G_{1}(s)}{\partial s} G_{2}(y) A_{1}(y) \cos (\rho t) d \rho d y d s \\
& B_{1}^{1}=\int_{r}^{R_{1}} \int_{0}^{s} \int_{0}^{\theta} G_{1}(r) G_{2}(s) \frac{G_{2}(s)}{s} G_{1}(y) A_{1}(y) \cos (\rho t) d \rho d y d s \\
& B_{1}^{2}=\int_{r}^{R_{1}} \int_{0}^{s} \int_{0}^{\theta} G_{1}(r) G_{2}(s) \frac{\partial G_{2}(s)}{\partial s} G_{1}(y) A_{1}(y) \cos (\rho t) d \rho d y d s \\
& B_{1}^{3}=\int_{r}^{R_{1}} \int_{s}^{R_{1}} \int_{0}^{\theta} G_{1}(r) G_{2}(s) \frac{G_{1}(s)}{s} G_{2}(y) A_{1}(y) \cos (\rho t) d \rho d y d s \\
& B_{1}^{4}=\int_{r}^{R_{1}} \int_{s}^{R_{1}} \int_{0}^{\theta} G_{1}(r) G_{2}(s) \frac{\partial G_{1}(s)}{\partial s} G_{2}(y) A_{1}(y) \cos (\rho t) d \rho d y d s \\
& A_{2}^{1}=\int_{0}^{r} \int_{0}^{s} \int_{0}^{\theta} G_{1}(s) G_{2}(r) \frac{G_{2}(s)}{s} G_{1}(y) A_{2}(y) \frac{\sin (\rho t)}{\rho} d \rho d y d s
\end{aligned}
$$




$$
\begin{aligned}
A_{2}^{2} & =\int_{0}^{r} \int_{0}^{s} \int_{0}^{\theta} G_{1}(s) G_{2}(r) \frac{\partial G_{2}(s)}{\partial s} G_{1}(y) A_{2}(y) \frac{\sin (\rho t)}{\rho} d \rho d y d s \\
A_{2}^{3} & =\int_{0}^{r} \int_{s}^{R_{1}} \int_{0}^{\theta} G_{1}(s) G_{2}(r) \frac{G_{1}(s)}{s} G_{2}(y) A_{2}(y) \frac{\sin (\rho t)}{\rho} d \rho d y d s \\
A_{2}^{4} & =\int_{0}^{r} \int_{s}^{R_{1}} \int_{0}^{\theta} G_{1}(s) G_{2}(r) G_{1}(s) G_{2}(y) A_{2}(y) \frac{\sin (\rho t)}{\rho} d \rho d y d s \\
B_{2}^{1}= & \int_{r}^{R_{1}} \int_{0}^{s} \int_{0}^{\theta} G_{1}(r) G_{2}(s) \frac{G_{2}(s)}{s} G_{1}(y) A_{2}(y) \frac{\sin (\rho t)}{\rho} d \rho d y d s \\
B_{2}^{2}= & \int_{r}^{R_{1}} \int_{0}^{s} \int_{0}^{\theta} G_{1}(r) G_{2}(s) \frac{\partial G_{2}(s)}{\partial s} G_{1}(y) A_{2}(y) \frac{\sin (\rho t)}{\rho} d \rho d y d s \\
B_{2}^{4}= & \int_{r}^{R_{1}} \int_{s}^{R_{1}} \int_{0}^{\theta} G_{1}(r) G_{2}(s) \frac{\partial G_{1}(s)}{\partial s} G_{2}(y) A_{2}(y) \frac{\sin (\rho t)}{\rho} d \rho d y d s \\
B_{2}^{3}= & \int_{r}^{R_{1}} \int_{0}^{R_{1}} G_{1}(r) G_{2}(s) \frac{G_{1}(s)}{s} G_{2}(y) A_{2}(y) \frac{\sin (\rho t)}{\rho} d \rho d y d s
\end{aligned}
$$

and

$$
\begin{aligned}
& G_{1}(x)=I_{0}\left(x \sqrt{\theta^{2}-\rho^{2}}\right) \\
& G_{2}(x)=K_{0}\left(x \sqrt{\theta^{2}-\rho^{2}}\right)-\frac{K_{0}\left(R_{1} \sqrt{\theta^{2}-\rho^{2}}\right)}{I_{0}\left(R_{1} \sqrt{\theta^{2}-\rho^{2}}\right)} I_{0}\left(x \sqrt{\theta^{2}-\rho^{2}}\right) \\
& A_{1}(y)=\left(H \frac{F r^{2}}{a S t}\right)\left\{\tau_{0} \sin \alpha \beta(y)\left[\frac{\beta(y)}{y}-2 \frac{\partial \beta(y)}{\partial y}\right]+\left[\frac{\partial^{2} P a}{\partial y^{2}}-\frac{1}{y} \frac{\partial P a}{\partial y}\right]\right\} \\
& A_{2}(y)=\left(\frac{H S t^{2}}{a}\right) \tau_{0} \cos \alpha \beta(y)\left[2 \frac{\partial \beta(y)}{\partial y}-\frac{\beta(y)}{y}\right]
\end{aligned}
$$

where $a=100$ and $H=1$.

\section{Acknowledgments}

We want to express our gratitude to the Department of Theoretical Physics of the University of Havana; to the colleagues of the IV Congress of Applied Mathematics in Vic, Barcelona, Spain, in September 1995; to colleagues of the University of Zaragoza and of UNED in Madrid, to Dr. P. Ripa from CICESE in Mexico, for a great interest in our work, and to Prof. A. Liñan from the Aeronautical Institute of Madrid, Spain, for fruitful discussions and comments. JMA wants to acknowledge the support of the Alma Mater grant to his research project.

\section{References}

[1] C.P. Jelesniansky, Monthly Weather Rev., 98, 472 (1970).

[2] B. Johns, Q. JLR. Met. Soc., 109, 211 (1983).

[3] C. B. Fandry, Dt. hidrogr Z 42, 307 (1989).

[4] P. Jerome, Coastal Engineering, 14, 1 (1990).

[5] S. R. Signorini, J. Geophys. Res., 97, 2229 (1992).

[6] A. M. Davies and J. E. Jones, Cont. Shelf Res., 12, 159 (1992). 
[7] R. W. Lardner, P. Smoczynski, Proc. R. Soc. of London A, 430, 263 (1990).

[8] R. W. Lardner, A. H. Al-Rabch, N. Gunay, Geophys. Res., 98, c10, 18229 (1993).

[9] N. S. Evsa, Meteor. I Guidrol., 3, 74 (1989).

[10] S. Pond and G. L. Pickard, Introductory Dynamical
Oceanography, Pergamon Press, Oxford (1983).

[11] B. Johns, Physical Oceanography of Coastal and Shelf Seas, Elsevier Publisher, Amsterdam (1983).

[12] A. Georgescu, Asymptotic Treatment of Differential Equations, Chapman \& Hall, London (1995). 